\title{
Application of improved DV-hop localization algorithm in port container positioning
}

\author{
Chen shuqian \\ Huaihai Institute of Technology \\ Huaihai Institute of Technology,HHIT \\ Lianyungang, China \\ chenshq@126.com
}

\author{
Zhang lihong \\ Huaihai Institute of Technology \\ Huaihai Institute of Technology,HHIT \\ Lianyungang, China \\ zlh2341@126.com
}

\begin{abstract}
In order to overcome the traditional positioning system has high cost, poor precision, demand for warehouse, container terminal management, using RSSI ( received single strength ) technology on the container for initial localization, then introduction of genetic DV-hop localization algorithm on the error correction. The simulation results show that improvement DV-hop algorithm improves the positioning accuracy of the node, reducing the positioning error, can make real-time monitoring for position, inventory and shift information of the goods, conducive to improving the efficiency of container management, more truly reflect the actual distribution of the containers.
\end{abstract}

Keywords-Containers; positioning; RSSI; genetic algorithm; DV-hop

\section{INTRODUCTION}

With the development of electronic and computer technology, modern logistics technologies become increasingly dependent on network technology, sensor technology and automation technology. With the popularity of RFID technology, its products prices fall and widely used in the logistics industry. In the traditional container tracking system, RFID technical help logistics enterprises and port to identify the container number, categories and other information, and through the network and database to the container site tracking, tracking of containers in the ports of loading and unloading and stacking, as well as the yard of the real-time scheduling and optimization, RFID technology can not provide accurate container real-time location information, using global positioning system (GPS) positioning system, sensor nodes can be precisely positioned, but the price is too expensive, can not be widely used in sensor node positioning. Wireless sensor combined with the container, can real-time detection of port container vacancy information, positioning and shifting of containers. Operators can optimize operations based on information provided by the system, improving the yard container management and efficiency in loading and unloading.

There are two kinds of nodes in wireless sensor networks: One is known of their physical location information, called anchor nodes, which are manually emplaced in port fixed position, and contains its own position information; another are not know their own physical location information, they determine its own position through the anchor node location information, the nodes are installed on the container, to provide real-time location information of container, called test node. Test node positioning algorithm is divided into two main types: independent of the distance and distance-related. Ranging technology of related to the distance positioning algorithm are RSSI, TOA, DOA, node position calculation methods include: Trilateral positioning algorithm, the triangulation method, least squares estimation positioning algorithm and so on, but the algorithm is relatively high hardware requirements. Sensor positioning technology based on distance-independent is the APIT positioning algorithms, DV-Hop algorithm and centroid localization algorithm, the algorithm has low power consumption, but the positioning accuracy is low [1,2]. For this reason, many scholars have proposed some improvements for wireless sensor node localization algorithms, effectively improve the localization accuracy, but there is a gap with the actual requirement.

\section{OVERVIEW OF NODE LOCALIZATION IN WIRELESS} SENSOR NETWORKS

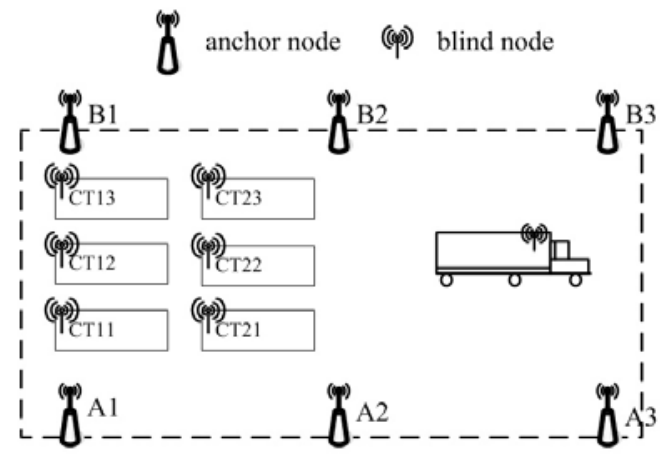

Figure 1 Distribution of nodes in wireless sensor networks

As the port container yard area restrictions, container stowage intensive, container node on a random, non-uniform distribution, which increase the difficulty of the container sensor node localization. If using GPS positioning each sensor node, expensive, high cost, not suitable for large-scale application; If use the manual positioning, long time consuming, low efficiency, also not suitable for large-scale application, therefore at present mainly uses sensor node localization technology. In wireless sensor networks, according to whether the known its position is divided into: the anchor nodes (the beacon nodes) and an unknown node. Small number of anchor nodes deployed in container yard, whose position is determined, on the container node is the 
unknown node, the greater number of the node, Unknown node is only through the use of surrounding or neighboring anchor node location, using a certain algorithm to get its own location information. As shown in figure 1.

\section{RSSI POSITIONING ALGORITHM}

RSSI is the received signal strength indicator, is a ranging technology using signal attenuation in the dissemination to speculate distance[3,4]. In general, relationship between wireless signal transmitting power and receiving power is shown below $[3,5]$.

$$
P_{\mathrm{r}}=P_{t} / d^{r}
$$

Where: $d$ is the distance between the transmitting and receiving nodes, $r$ is propagation factor, its value depends on the wireless signal propagation environment.

Take the logarithm on both sides of Equation (1),

$P L(d)=A_{0}-10 \cdot r \lg d$

Where: $A_{0}=10 \lg P_{t}$ is the received signal power in the signal propagation $1 \mathrm{~m}$ far, $P L(d)=10 \lg P_{r}$ is the received signal strength from the transmitter point $d \mathrm{~m}$, the RSSI value. From equation (2) can be seen, the value of the constants $A_{0}$ and $r$ determine the relationship between RSSI value and the signal propagation distance. In theory, you can use triangular measurements to determine the spatial location of an unknown node by four anchor nodes RSSI information. In this article apply to the vector product is $X=\left(x_{1} y_{1} z_{1}, x_{2} y_{2} z_{2}, \cdots, x_{n} y_{n} z_{n}\right)$, simulation of random randomly placed to put the initial position of the container sensor nodes in the $R^{3}$ space network topological region. Assume that $\mathrm{m}$ anchor node locations are known, $n-m$ is the unknown number of unknown node, Network location is $\left(x_{m+1} y_{m+1} z_{m+1}, x_{m+2} y_{m+2} z_{m+2}, \cdots, x_{n} y_{n} z_{n}\right)$, the node localization problem can be described as, uses of known location of anchor nodes, through the geometric theory to solve the unknown node's coordinates, which is arranged in the area of the network with communication constraints. In the wireless sensor network positioning process, the case of four anchor nodes and unknown node do not exist any errors, the distance formula is as follows[6]:

$$
\left\{\begin{array}{l}
\left(x_{1}-x\right)^{2}+\left(y_{1}-y\right)^{2}+\left(z_{1}-z\right)^{2}=d_{1}^{2} \\
\left(x_{2}-x\right)^{2}+\left(y_{2}-y\right)^{2}+\left(z_{2}-z\right)^{2}=d_{2}^{2} \\
\left(x_{3}-x\right)^{2}+\left(y_{3}-y\right)^{2}+\left(z_{3}-z\right)^{2}=d_{3}^{2} \\
\left(x_{4}-x\right)^{2}+\left(y_{4}-y\right)^{2}+\left(z_{4}-z\right)^{2}=d_{4}^{2}
\end{array}\right.
$$

Where $(x, y)$ represents the Euclidean distance between the unknown node coordinate values and the known four nodes, combined with equation (2), in the region of the unknown node, using the Taylor series expansion formula to take the first-order partial derivatives, that is:

$$
\begin{aligned}
& d=d(x, y)+\frac{\partial d}{\partial x}\left(x_{1}-x\right)+\frac{\partial d}{\partial y}\left(y_{1}-y\right)+\frac{\partial d}{\partial z}\left(z_{1}-z\right) \\
& \text { So, } f(x)=\frac{1}{\sqrt{2 \pi} \delta} e^{\frac{d(x, y)+\frac{\partial d}{\partial x}\left(x_{1}-x\right)+\frac{\partial d}{\partial y}\left(y_{1}-y\right)+\frac{\partial d}{\partial z}\left(z_{1}-z\right)}{2 \delta^{2}}}
\end{aligned}
$$

Where $\delta=\sqrt{\sum_{i=1}^{k}\left(x_{i}-\mu\right) / k-1}, \mu$ is a Gaussian

distribution in the Gaussian model, $\mu=\sum x_{i} / k$. Determine the spatial plane geometry relation[7].

\section{DV-HOP LOCALIZATION ALGORITHM}

In the actual application of container port, as the container obstructions to the signal attenuation, multipath effects caused by box metal surface refraction and reflection, will give a great deal of RSSI positioning error. For the mutual blocking of container, surface reflectance between metal box and other factors, on the basis of the original RSSI positioning, use the DV-Hop model assisted positioning, improve positioning accuracy. DV-Hop is proposed by American scholar Dragos Niculescu .DV-Hop is a location algorithm without ranging, which use Vector routing and GPS positioning principle. By measuring the distribution of the minimum number of hops of the unknown nodes and anchor nodes, and use the jump distance instead of the actual distance to estimate the unknown node location coordinates to determine node location. DV-Hop algorithm node localization process specifically as follows:

\section{A. Distance vector exchange}

Anchor nodes broadcast data packet to the neighbor nodes using flooding, unknown node obtains the minimum hop count between each anchor node, so that each unknown node contains a table $\left\{x_{i}, y_{i}, n_{i}\right\}$, Which $\left\{x_{i}, y_{i}\right\}$ is the location of the anchor node, $n_{i}$ is the smallest hop count between this node and anchor node $\mathrm{i}$. Because each node may receive multiple broadcast packets of the same anchor node, then the point uses the minimum hop count data packet to replace other packets. Finally, each wireless sensor nodes will collect all the anchor node position information and the minimum hop count from anchor node[8].

\section{B. Hop distance calculation and broadcasting}

In the yard, after fixed anchor nodes obtain the location information of other container node and separated by the number of hops and the number of separated hops, can use formula (6) estimated the average hop distance, then anchor nodes will make the value as a correction broadcast to the network, unknown node receives the average hop distance, in order to calculate the distance to each anchor node.

$$
c_{i}=\frac{\sum_{j \neq i} \sqrt{\left(x_{i}-x_{j}\right)^{2}+\left(y_{i}-y_{j}\right)^{2}}}{\sum h_{i}}
$$

Where $h_{i}$ represents the number of hops between this anchor node to anchor node, $\left(x_{j}, y_{j}\right)$ represents the coordinates of the other anchor nodes.

\section{The calculation of the sensor node location}

When the unknown node on the container obtained more than four fixed anchor node distance, we can use triangular measurements to determine the location of the sensor nodes. 
The location of containers stacked in the yard is uncertain, leading to the sensor node distribution is uneven. Estimation of the hop count by the distance vector exchange protocol is the average hop distance of the whole sensor network, therefore, the calculated distances between nodes has larger error to the actual distance.

\section{IMPROVE THE SENSOR NODE LOCALIZATION ALGORITHM}

For the shortcomings of the above algorithm location error is large, many scholars have proposed improved algorithm, but the result is not satisfactory, can not effectively improve the positioning accuracy of sensor nodes, and thus cannot be accurately determine the location of containers. In this paper, on the basis of without adding any node, use a genetic algorithm on node positioning error correction, thereby improving the positioning accuracy of sensor nodes.

1) Initialize the population. Assuming in the network the number of unknown node is $\mathrm{m}$ and the number of anchor nodes is $\mathrm{n}$, unknown node coordinates is $\left(x_{1}, y_{1}\right), \cdots,\left(x_{j}, y_{j}\right), j=1,2,3, \cdots, m$, anchor nodes is $\left(a_{1}, b_{1}\right), \cdots,\left(a_{j}, b_{j}\right), j=1,2,3, \cdots, n$, each unknown node can communicate with four anchor nodes, this randomly generated ${ }^{q}$ initial population.

2) Set the fitness function. Through the genetic algorithm to optimize DV-Hop positioning algorithm, the optimization goal is to improve the positioning precision of sensor node, therefore using the following formula as fitness function:

$$
f\left(x_{j}, y_{j}\right)=\min \left(\sum_{j=1}^{k} \sqrt{\left(x_{j}-a_{i}\right)^{2}+\left(y_{j}-b_{i}\right)^{2}}-d_{i}^{2}\right)
$$

Where, $\left(a_{j}, b_{j}\right)$ represents the $i$ th anchor node $i,\left(x_{j}, y_{j}\right)$ represents the $j$ th unknown sensor nodes.

We can see from equation (7), fitness value of optimal individual is the optimal estimation position of the unknown node.

3) Genetic manipulation. Adopt the basic roulette selection operator, adaptive way crossover and mutation operators, in the early stage of the population evolution, probability of crossover and mutation is corresponding larger, passed on to the late evolution, crossover and mutation probability gradually decreases, this can speed up the population rate and improve the search speed of the optimal solution, at the same time to prevent the appearance of local optimum solution.

4) Algorithm end conditions. When the evolution of algebra achieved the maximum algebraic or groups of solutions achieved optimal, the algorithm ends.

\section{SIMULATION EXPERIMENT}

This paper uses the MATLAB7.0 language simulation testing laboratory, compare the algorithm with the DV-Hop algorithm. Randomly generated the coordinates of the anchor nodes and unknown nodes, sensing area size is $300 \mathrm{~m} \times$ $300 \mathrm{~m}$. Algorithm evaluation standard is the average localization error, carrying out 20 time of simulation experiment on the above two algorithms, improved DV-Hop algorithm and the DV-Hop algorithm use the average of the simulation results as the final positioning accuracy value.

\section{A. anchor node number effects on positioning error}

Improved DV-Hop algorithm and traditional DV-Hop algorithm's average positioning error and proportion of anchor nodes change curve are shown in Figure 2. We can see from Figure 2, decreased with an increasing share of anchor nodes, the positioning error of the two algorithms is decreased, but average location error of RSSI positioning combined with improved DV-Hop algorithm is lower about 9\% than traditional DV-Hop algorithm, and compared with the traditional DV-Hop algorithm positioning error decreases quickly. The comparison results show that the RSSI positioning combined with improved DV-Hop algorithm reduce the average location error value, improved the situation of the positioning deviation caused by Metals and obstructions.

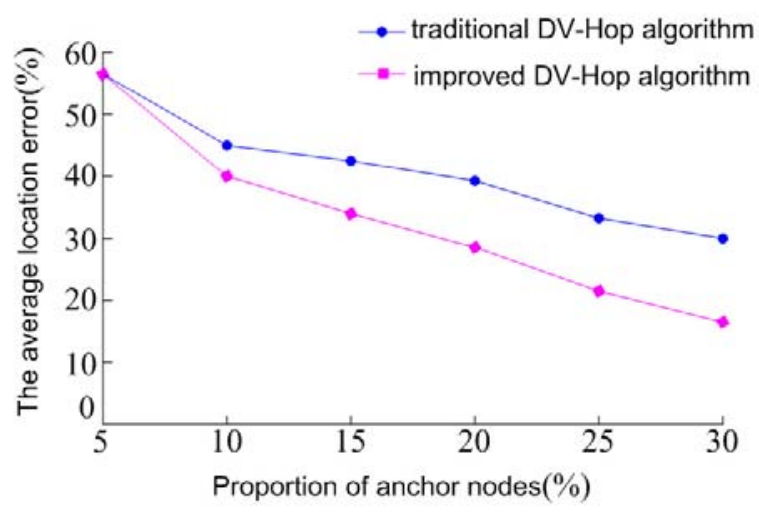

Figure 2 Anchor nodes change impacts on average location error

\section{B. Number of points impact on the average positioning accuracy}

In the case of the same number of anchor nodes, the total number of nodes increases, the average positioning accuracy curve of RSSI positioning combined with improved DV-Hop algorithm and traditional DV-Hop algorithm are shown in Figure 3. We can see from the comparison of the results in Figure 3, with the total number of nodes in sensor networks increases, the average positioning accuracy of the two algorithms have been improved, RSSI positioning combined with improved DV-Hop algorithm improve even more sharply, more instructions, using RSSI localization combined with genetic algorithm to improve the DV-Hop location algorithm is effective, able to adapt to the use of container positioning of the port, improve the utilization of the port yard. 


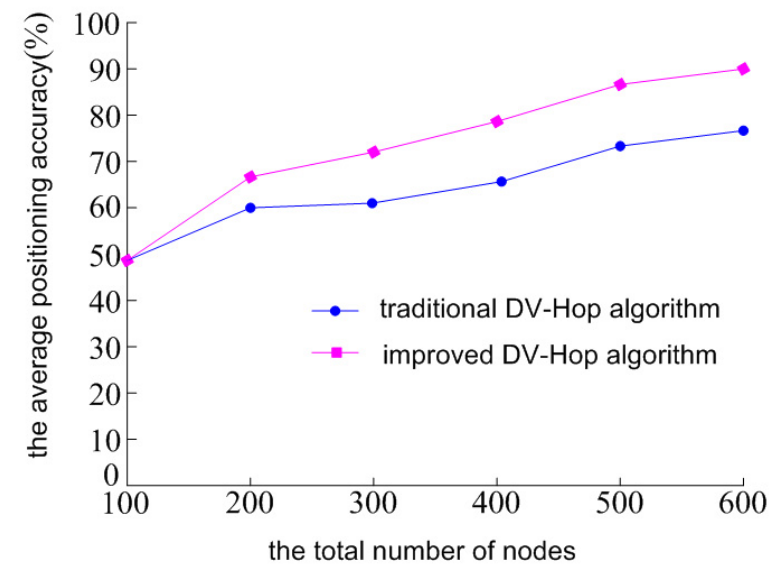

Figure 3 The total number of nodes change impact on the average positioning accuracy

\section{CONCLUSION}

This paper presents the RSSI positioning combined with improved DV-Hop sensor node localization algorithms, the genetic algorithm is introduced to positioning error correction, provides more accurate container positioning scheme, to solve the problems of port storage yard utilization rate, using wireless sensor networks to locate the container, using sensor nodes effectively increasing the effectiveness of port container collect information and real-time, provide a reliable basis for decision-making of the intelligent port production scheduling. Comparison of simulation experimental results showed that this method of positioning accuracy has met the demand of the port.

\section{REFERENCES}

[1] Mao Yongyi,Li Mingyuan, and Zhang Baojun “A TOA/ AOA Location Algorithm in NLOS Environment, J Journal of Electronics \& Information Technology. 2009,31(1),pp.37-41

[2] Zhang Jing,Cao Dun, and Fu Ming, "Improvement on location error and coverage rate in DV-Hop algorithm," Journal of Computer Applications. 2011,31(7),pp.1944-1947

[3] Zhan Jie,Wu Lingxi,and Tang Zhijun, "Ranging Method and Accuracy Analysis Based on RSSI of Wireless Sensor Network,” Telecommunication Engineering . 2010,50(4),pp.83-87

[4] Zhang Jianwu, Zhang Lu, and Ying Ying, "Research on Distance Measurement Based on RSSI of ZigBee,” Chinese Journal of Sensors and Actuators, 2009.22(2), pp.285-288.

[5] Ren Zhi,LiI Qingyang, and Chen Qianbin, "Modeling and simulation of fading and loss for wireless networks," Systems Engineering and Electronics . 2009.31(12),pp.2813-2819.

[6] Chen Sanfeng,Chen Wanming, "Localization algorithm based on analysis of RSSI error for Wireless Sensor Networks ,' Computer Engineering and Applications. 2011.74(14),pp10-12.

[7] Zhan Jijun,Li Hua, and Sun xiang, "Research on the signal random attenuation coefficient based on RSSI in WSN localization technology," $[\mathrm{c}] .5^{\text {th }}$ International Conference on Wireless Communications, Networking and Mobile Computer,2009,pp.1-4

[8] Ma'roml F G ,Pe'rez G M, “Security threats scenarios in trust and reputation models for distributed systems,”Computer \& Security,2009, 28(1),pp.545-556 